\title{
Evolution of Smart Buildings
}

\author{
Gabriel Iulian Fântână \\ Department of System Theory \\ "Polytechnic" University Bucharest \\ Bucharest, Romania
}

\author{
Stefan Adrian Oae \\ Department of Engineering and Technological Systems \\ Management, "Polytechnic" University Bucharest \\ Bucharest, Romania
}

Received: January 19, 2021. Revised: March 10, 2021. Accepted: March 13, 2021. Published: March 23, 2021.

\begin{abstract}
Today, in times of economic crisis, the need for optimization is more present than ever. Since buildings are responsible for almost $50 \%$ from total environment pollution, which means a significant resource spending, changes for improvement must be done.
\end{abstract}

Keywords—smart, building, sustainability, integration, energy, management.

\section{INTRODUCTION}

In every country in the world, the built environment normally constitutes more than half of the total national capital investment and construction represents only $10 \%$ of GNP. [1]. If this value will be translated into energy consumption which defines the quality of life, two things will emerge: the enormous pollution and the amount of spent resources.

Clean air is a mixture of gases - $78 \%$ nitrogen and $21 \%$ oxygen - with traces of water vapor, carbon dioxide, argon, and various other components.

In Europe only, air pollution from the 10,000 largest polluting facilities, costs citizens between 102 and 169 billion $€$ in 2009 . Half of the total damage cost (between 51 and 85 billion $€$ ) was caused by just 191 facilities [2].

The industrial facilities considered include large power plants, refineries, manufacturing combustion and industrial processes, waste and certain agricultural activities. Emissions from power plants contributed the largest share of the damage costs (estimated at $66-112$ billion $€$ ). Other significant contributions to the overall damage costs came from production processes (23-28 billion $€$ ) and manufacturing combustion (8-21 billion $€$ ). Sectors excluded from this analysis are transport, households and most agricultural activities - if these were included the cost of pollution would be even higher.

The tall buildings were meant to exploit the land but have the negative effects in the environment and create new problems including increasing congestion population, pollution, reduce citizen access to fresh air and sunlight.

In actual tendencies of population increasing and land shortage, tall buildings cannot be replaced.

In the US, according to the Department of Energy, buildings consume approximately $37 \%$ of the energy and $68 \%$ of the electricity produced annually. Energy-management practices and energy-efficient equipment can reduce energy costs by at least $20 \%$ - a net savings opportunity worth more than 11 billion $\$$ by 2010 [3].
In Eastern Europe, Romania is the first country that will fulfill Kyoto objectives by reducing its greenhouse gas by $8 \%$. The $\mathrm{CO}_{2}$ emission is also $58 \%$ less than reference and this was possible because of reduction of burned fossil fuel [4].

Pollution prevention is the "gateway" to sustainability and also a waste. Understanding how is generated and how can be minimized is the first step to eliminating it and increase efficiency and develop sustainable production methods.

Sustainable design is not just about cost; it offers economic, environmental and social benefits. The solar panels, for instance, can reduce the amount of natural resources spent and a general lower cost for used energy. This also helps by reducing the load on local energy distribution stations, so the "black-out" phenomena can be easier avoided.

\section{SMART BUILDING EVOLUTION}

Born in early 80 's, the smart building concept was involving especially an extensive use of elaborate centralized electronic systems to make possible the control of building support and communication systems for voice and data. The initial stage promoted communication networks in order to centralize word processing services and limited interaction between occupants and BAS though an easy user interface such as touch switches.

Intelligent buildings use technological solutions to improve the building environment and functionality for occupants/tenants while monitoring costs. Improving end user security, comfort and accessibility will enhance user's productivity and comfort levels. The owner/operator wants to provide optimal functionality while reducing individual costs. An effective energy management system, for example, provides lowest cost energy, avoids waste of energy by managing occupied space, and makes efficient use of staff through centralized control and integrating information from different sources.

There are many definitions that were developed but the most accepted one is formulated by Cerdà institute in Barcelona [5]: "system that support the flow of information throughout the building, offering advanced services of business automation and telecommunications, allowing furthermore automatic control, monitoring management and maintenance of the different subsystems or services of the building in an optimum and integrated way, local and/or remote, and designed with sufficient flexibility to make possible in a simple and economical way the implementation of future systems." 
An Intelligent Building is one which provides an efficient and cost-effective environment through optimization of its four basic elements [6]:

\section{Smart Building Basic Elements}
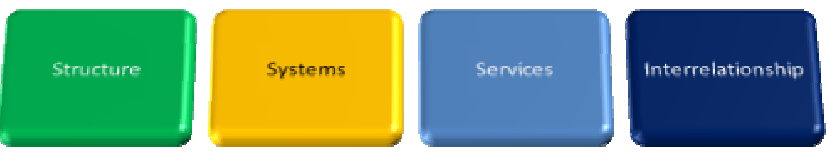

Fig 1 Smart building's main four elements

In its early stages, the concept was referring more to automation than intelligence. The main difference between these two was that the first one use only the component systems and provide their optimal behavior, while the second one deals specially with mathematical and computers sciences which, by using of extended algorithms, can "think" and dynamically change parameters in order to anticipate and optimize occupant's expectations.

The history of IB can be divided into several stages: during 60 's, the smart or intelligent building had been equipped with building automation system that had capabilities as "work saving time' device; then, first energy crisis in 1973 led people to reexamine the ways of energy use and generation awareness on energy conservation, so the smart features had become associated with energy conservation and crime prevention systems; integrated single function/dedicated systems (80's);integrated multifunction and building level systems (90's); computer integrated building (2000); enterprise network integrated systems (today).

Fig 2 Smart building evolution through time

Integrated single function/dedicated systems, all the BA subsystems (including security control; access control;

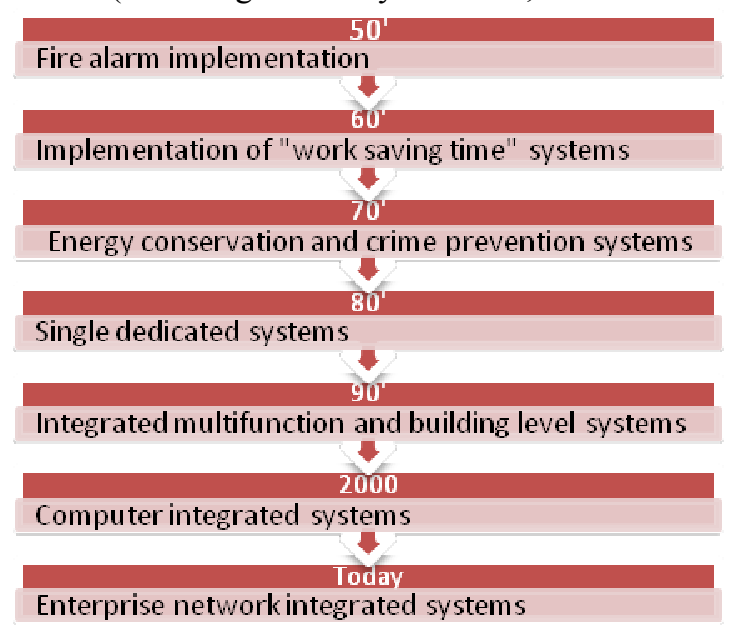

heating, ventilation and air - conditioning [HVAC] control; lighting control; lift control; other electrical systems; fire automation; etc.) and CA subsystems (including electronic data processing and data communication; telefax and text communication; voice communication; $\mathrm{TV}$ and image communication; etc.) were integrated at the level of a single or individual function subsystem. Integration and communication between the automation systems of different subsystems was impossible.

Integrated multifunction systems provided integrated security, access control, and automation and/or services systems. Also were unified networks for text, data, voice and multimedia communications.

Building level integrated systems had both BA and communication systems integrated at building level as building automation system (BAS) and integrated communication system (ICS). At this stage, a BA system could be accessed remotely using a modem, while the cellular phone for voice and data communication was introduced.

Computer integrated building, made available various types of networks became available that were used in practice progressively, by the use of Internet protocol (IP) network technologies and increased network capacity. Remote monitoring and control could be achieved via the Internet.

Enterprise network integrated system promotes the intelligent systems that can be integrated and managed at domestic, enterprise or city level. Intelligent building systems are not enclosed within buildings anymore but connected with IB systems in separate buildings via the global Internet infrastructure. Combining BAS and IT through the backbone of an Internet Protocol network allows multiple services to be delivered to occupants.

Integration and management at this level become possible due to the applications of modern IT technologies such as Web Services, XML, remote portfolio management and helpdesk management, among others. In terms of communication, multimedia communication via cellular phone has been brought into practical use.

\section{CONCLUSIONS}

The integration of all components and subsystems has been the main focus of IB technology development. Integration is essential for most functions, such as automatic monitoring and management, building performance optimization and diagnosis. Function integration increases the flexibility and possibilities of intelligent management of buildings. Digital technology is a key component into the integration because traditional ones still have many constraints in terms of information exchange and integration. The microprocessor proves amazing power in computation, transmitting and processing information, being the key element of digital systems and of IB and BA systems.

Modern building systems have been becoming very large and complex in terms of system scale, hardware and software system configurations, while their functions and capacities have been increasing progressively [7]. As can be seen in fig.3, the plug loads increased from 1995 and prognosis is, at least, frightening regarding its evolution till 2035 [8]. 
Electricity use breakdown for commercial buildings

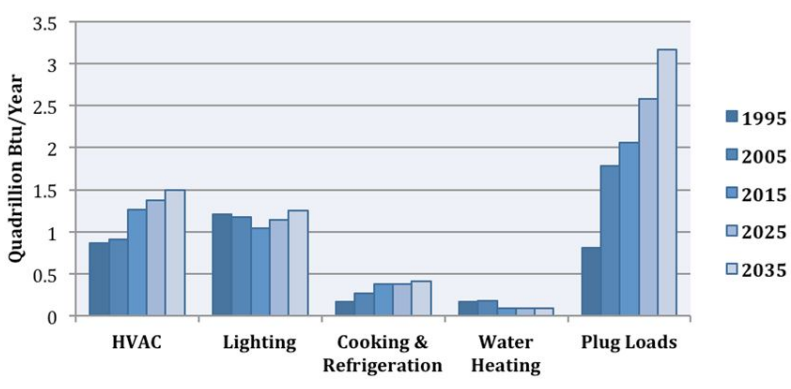

Fig 3 Plug loads evolution between 1995 and 2035

Plug loads are a major provider to building energy consumption, especially in offices. In commercial buildings, plug loads are one of the fastest-growing end uses in terms of energy consumption and typically account for 30-35 percent of the total electricity used [9].

In order to optimize costs, many commercial building owners and managers have installed smart building energy management systems in order to reduce energy use and operating expenses.

Today, the automated, or smart, building energy management systems market is to almost quadruple in size and be worth over $\$ 1$ billion by 2020 [10].

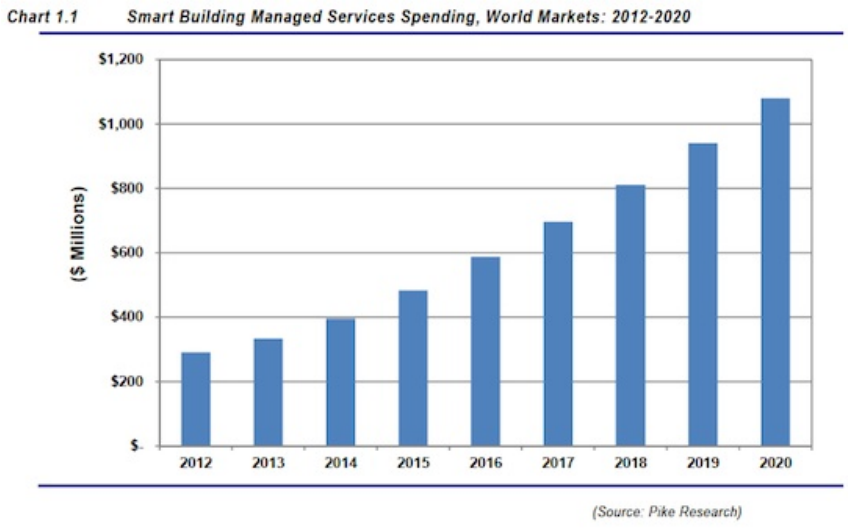

Fig 4 Smart Building Managed Services Spending [10]

Pike puts the market potential for all buildings in the U.S. at more than $\$ 37$ billion in 2010. By 2020, the prediction is that the market to be worth more than $\$ 45$ billion. [10]

System reliability is an important issue also. Developing a decentralized network or a local area network (LAN) is the key will solve the system reliability issues and simplify IB networks. Distributed intelligence is a major optimal solution to ensure connection through complex IB and BA systems. 'Integrated but independent' is one of the most essential concerns in the development and configuration of these systems.
Chart 1.1 Total Potential Market for EMS by Building Usage, United States: 2010-2020

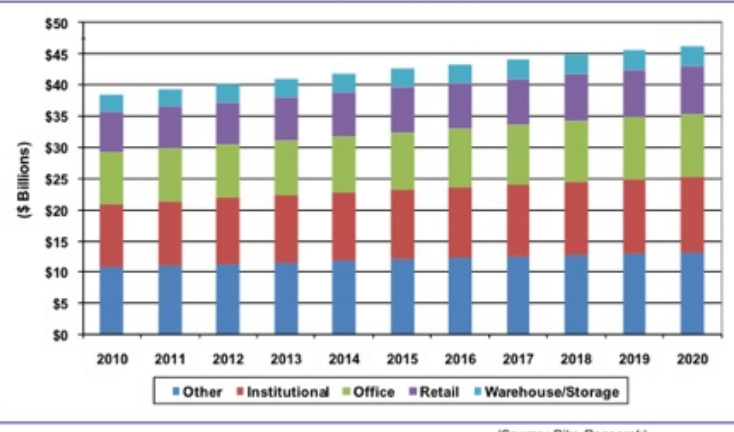

(Source: Pike Resoarch)

Fig 5 Total Potential Market for Energy Management Systems by Building Usage [10]

Intelligent buildings need to be sustainable (i.e. sustain their performance for future generations), healthy and technologically up to date; meet regulatory demands; meet the needs of the occupants; and be flexible and adaptable enough to deal with change. Buildings will contain a variety of systems devised by many people, and yet the relationship between buildings and people can only work satisfactorily if there is integration between the supply and demand-side stakeholders as well as between the occupants, the systems and the building. To achieve this, systems' thinking is essential in planning, design and management, together with the ability to create and innovate while remaining practical. The ultimate objective should be simplicity rather than complexity. This requires not only technical ability but also the powers of interpretation, imagination and even intuition. Building Regulations can diminish creativity but are necessary to set a minimum level of expectation and obey health and safety requirements. However, we should aim at designing well above these conditions. After all, buildings form our architectural landscape and the environment they generate, should uplift the soul and the spirit of those people within them as well as those who pass by them.

\section{REFERENCES}

[1] CICA (2002). Construction. UK, Beacon Press.

[2] European Environment Agency, Kongens Nytorv 61050 Copenhagen K Denmark, "Revealing the costs of air pollution from industrial facilities in Europe - a summary for policymakers," EEA Technical report No $15 / 2011$.

[3] McKinsey, "Reducing US GHG Emissions: How much at What Cost?" US GHG Abatement Mapping Initiative

[4] Gaman (Pasvantu) Irina Cristina, Triunghiul Dezvoltarii Durabile(Economic, Mediu, Social)

[5] Lafontaine, J. (1999). Intelligent building concept. Ontario: EMCS Engineering Inc.

[6] Wang Shengwey, Ïntelligent Building and Building Automation”, Spon Press, USA, 2010.

[7] Wong, J. K. W., Li, H. and Wang, S. W. (2005) 'Intelligent building research: a review',Automation in Construction, 14(1): 143-59.

[8] Barnaby Chambers, "Graphic based on data from the Energy Information Administrators", Annual Energy Outlook 2012

[9] Tolga Tutar, "How to defeat the massive plug-load monster", greenbiz.com, May 16, 2012.

[10] Navigantresearch.com, "Energy Management Systems for Commercial Buildings", november 18, 2009.

Creative Commons Attribution License 4.0 (Attribution 4.0 International, CC BY 4.0)

This article is published under the terms of the Creative Commons Attribution License 4.0

https://creativecommons.org/licenses/by/4.0/deed.en_US 